\title{
New exploration of the treatment of nontuberculous mycobacterium lung disease: Moxifloxacin may be a first-line drug for the treatment of NTM-PD
}

\section{Binbin Feng}

Shandong Public Health Clinical Center

Meiying Lin

Shandong Public Health Clinical Center

Qinghu Yan

Shandong Public Health Clinical Center

Lihua Qiu ( $\sim 13969085108 @ 163 . c o m$ )

Shandong Public Health Clinical Center

\section{Research Article}

Keywords: moxifloxacin, nontuberculous mycobacterium, nontuberculous mycobacterium lung disease, Mycobacterium intracellulare

Posted Date: February 23rd, 2022

DOI: https://doi.org/10.21203/rs.3.rs-1272399/v1

License: () (i) This work is licensed under a Creative Commons Attribution 4.0 International License. Read Full License 


\section{Abstract}

Objective: By comparing the results of moxifloxacin sensitivity test and clinical efficacy in nontuberculous mycobacterium lung disease (NTM-PD) further confirm the necessity of moxifloxacin as a first-line drug for the

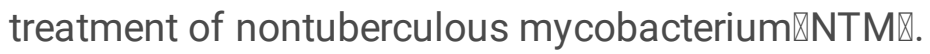

Method: Positive samples of sputum, bronchoscope brush or alveolar lavage fluid cultured in Bactec MGIT960 system were retrospectively selected from inpatients in Shandong Provincial Chest Hospital, China from January 2015 to December 2020. The strains were identified by MPB64 colloidal gold method, gene chip and microbial mass spectrum identification system. The drug detection of NTM was performed by microplate method, Meanwhile, the efficacy of moxifloxacin in NTM-PD treatment regimen was compared.

Results: 1 . Among the 575 patients with positive sample culture, the bacteria species of 485 patients were identified. A total of 19 species of NTM were detected, and the majority of them were Mycobacterium intracellulare $(68.87 \%, 334 / 485)$.2. The results of microplate assay of 219 NTM patients showed that all NTM strains were highly sensitive to moxifloxacin, amikacin and clarithromycin $[100.00 \%(219 / 219), 96.80 \%$ (212/219), $94.52 \%(207 / 219)$, respectively]. 3. This study showed that the bacteriological cure rate of the NTMPD treatment regimen containing moxifloxacin was $73.68 \%$ (42/57), significantly higher than that of the regimen without moxifloxacin $(55.45 \%, 56 / 101)$, and the difference was statistically significant $(P<0.05)$.

Conclusion: In this study, all the strains of NTM patients were $100 \%$ sensitive to moxifloxacin, and the clinical bacteriological cure rate of moxifloxacin containing regimen was significantly improved. Therefore, moxifloxacin is recommended as the first-line drug for the treatment of NTM.

\section{Introduction}

Nontuberculous mycobacteria (NTM) is a mycobacteria, except mycobacterium tuberculosis (MTB) complex and the leprosy mycobacteria, in the genus Mycobacteria. Widely distributed in nature, there are more than 190 known NTMs ${ }^{[1]}$. NTM exists widely in the natural environment such as water, soil, dust, etc. It can be infected by humans and certain animals, mainly causing lung diseases. Data ${ }^{[203]}$ shows that the incidence and prevalence of NTM disease are increasing in several countries and regions, and even exceed the incidence and prevalence of tuberculosis. At present, NTM-PD is difficult to treat. Most of NTM-PD have a natural resistance to anti-tuberculosis drugs, limited availability of drugs, a poor therapeutic effect, high failure rate and high recurrence rate. Therefore, it is of great significance to understand the drug sensitivity of NTM-PD and choose effective drug therapy. The author retrospectively studied the drug sensitivity and clinical treatment of NTM-PD patients hospitalized in Shandong Provincial Chest Hospital, and found that moxifloxacin was generally sensitive to NTM strains, and the treatment effect of the program containing moxifloxacin was better than that without moxifloxacin, providing a reliable scientific basis for the first-line effective drugs for the treatment of NTM-PD.

\section{Subject And Method}

1囚Research Subjects: A total of 575 patients with positive sputum, bronchoscope brush or alveolar lavage fluid were retrospectively selected from the patients in Shandong Provincial Chest Hospital from January 2015 to 
December 2020. The drug results of 219 samples of patients who underwent drug sensitivity test were analyzed. At the same time, the efficacy of moxifloxacin in NTM-PD treatment regimen were compared.

2. Inclusion criteria: Inclusion criteria are referred to as Guidelines for the Diagnosis and Treatment of Nontuberculous Mycobacteria Disease (2020 Edition) ${ }^{[4]}$, and meet the following 2 criteria,

1هDiagnostic criteria for NTM-PD: The patient has respiratory symptoms and/or systemic symptoms, and the chest imaging examination has found cavitation opacities, multifocal bronchiectasis and multiple micronodular lesions, etc. Other diseases have been ruled out. The diagnosis of NTM-PD can be made if one of the following conditions is met: (1) Two sputum specimens separately sent for examination were cultured positive for NTM and identified as the same pathogen, And/or NTM molecular biological detection were the same pathogenic bacteria; (2) The NTM culture and/or molecular biological test of bronchial rinsing fluid or bronchoalveolar lavage fluid were positive once.

$2 \varangle$ Patients who accord with one of the following conditions in the treatment outcome:(1) Bacteriological cure: After three consecutive NTM culture negative sputum with at least 1 month interval, and one negative NTM culture of bronchial rinsing fluid or lavage fluid in the absence of sputum, several more consecutive NTM cultures were negative until the end of anti-mycobacterium therapy. (2) Treatment failure: Anti-mycobacterium treatment $\geq 12$ months, After the bacteriology negative turn, the patients reappear to cultivate the same kind of NTM 2 and above positive or cultivation has been unable to turn negative.

3. Exclusion criteria: 1) Less than 12 months of anti-NTM treatment; 2) Patients lost during treatment.

4. Research Method

4.1 Strain Identification: (1) The laboratory of the hospital shall operate in accordance with the Testing Procedures for Tuberculosis Diagnostic Laboratory, and use the MPB64 antigen to conduct preliminary identification to the culture-positive strain. When the MPB64 antigen test was negative, NTM was initially identified. (2) 17 species of mycobacteria (including MTB complex, M.intracellulare, M.kansassi, M.avium, M.abscessus, M.fortuitum, etc.) use gene chip detection (DNA microarray chip method) for bacterial species identification. The operation is carried out in strict accordance with the instructions: Nucleic acid extraction, PCR amplification, microarray hybridization, microarray washing and drying, microarray scanning and result interpretation. (3) The laboratory used the matrix assisted laser desorption ionization time of flight mass spectrometry microorganism identification system to identify mycobacteria species other than 17 species according to the manufacturer's instructions.

4.2 Drug Sensitivity test: The liquid-based microporous plate method ${ }^{[5]}$ was used to carry out the NTM drug sensitivity test of 15 kinds of drugs in the logarithmic growth period, and the ultrasonic turbidimetry of bacteria was carried out by using the bacterial ultrasonic dispersion counter. The operation interpretation was carried out according to the instructions of NTM drug sensitivity test kit (microplate method). The minimum inhibitory concentration (MIC) of different drugs to determine drug resistance are: Linezolid: $\geqq 32 \mu \mathrm{g} / \mathrm{mL}$; Clarithromycin: $\geqq 16 \mu \mathrm{g} / \mathrm{ml}$; Rifampicin: $\geqq 6 \mu \mathrm{g} / \mathrm{ml}$; Amikacin: $\geqq 64 \mu \mathrm{g} / \mathrm{ml}$; Imipenem: $\geqq 16 \mu \mathrm{g} / \mathrm{ml}$; Ethambutol: $\geqq 5 \mu \mathrm{g} / \mathrm{ml}$; Cefoxitin: $\geqq 80 \mu \mathrm{g} / \mathrm{ml}$; Azithromycin: $\geqq 16 \mu \mathrm{g} / \mathrm{ml}$; Rifabutin: $\geqq 2 \mu \mathrm{g} / \mathrm{ml}$; 
Tobramycin: $\geqq 6 \mu \mathrm{g} / \mathrm{ml}$; Gatifloxacin: $\geqq 1 \mu \mathrm{g} / \mathrm{ml}$; Moxifloxacin: $\geqq 8 \mu \mathrm{g} / \mathrm{ml}$; Doxycycline: $\geqq 8 \mu \mathrm{g} / \mathrm{ml}$; Minocycline: $\geqq 8 \mu \mathrm{g} / \mathrm{ml}$; Compound Sulfamethoxazole: $\geqq 80 \mu \mathrm{g} / \mathrm{ml}$.

4.3 Treatment plan evaluation: The bacteriological cure rates of the moxifloxacin-containing regimen and the moxifloxacin-free regimen were investigated and analyzed.

5.Statistical Processing: Data were collected by Microsoft Excel 2016 and analyzed by SPSS19.0 software to perform statistical analysis of the data. A Koimogorov-Smirnov test was used to examine whether the data obeyed normal distribution. Data obeying normal distribution were presented as mean \pm standard difference $(\bar{X} \pm s)$. An independent sample t test was used for continuous variables that obeyed normal distribution Comparison of reaction rates between the two groups was per-formed using a chi-square test. Value of $P<0.05$ was considered indicative of statistically significant publication bias.

\section{Results}

1.Basic information of patients in two groups $₫ A$ total of 57 patients who received the moxifloxacin-containing

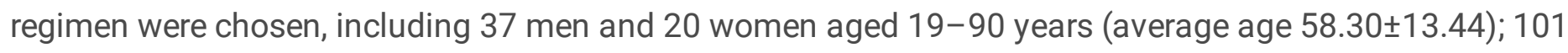
patients who received the moxifloxacin-free regimen were chosen, including 64 men and 37 women aged 1890 years (average age $54.49 \pm 16.81$ ); A comparison of basic clinical information between the groups showed no statistically significant differences $(P>0.05)$.

2.NTM strain identification distribution: 484 of 575 NTM patients were identified by gene chip and microbial mass spectrum identification system, and 19 different bacterial infections were detected. The main strains were M.intracellulare $₫ 68.87 \% \varangle 334 / 485 \rrbracket$, M.kansassi $₫ 12.16 \% \varangle 59 / 485 \rrbracket, M$. abscessus $\varangle 8.25 \% \varangle 40 / 485 \rrbracket$. (See Table 1 for details).

Table 1 Strain distribution in NTM-PD patients hospitalized from 2015 to 2020 


\begin{tabular}{|c|c|c|c|c|c|c|c|c|c|}
\hline NTM specles & 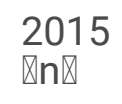 & $\begin{array}{l}2016 \\
\bigotimes \mathrm{n} \rrbracket\end{array}$ & 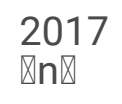 & 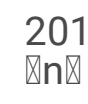 & & $\begin{array}{l}2019 \\
\bigotimes \mathbf{n} \rrbracket\end{array}$ & $\begin{array}{l}2020 \\
\bigotimes \mathrm{n} \rrbracket\end{array}$ & $\begin{array}{l}\text { total } \\
\bigotimes \mathrm{n} \rrbracket\end{array}$ & $\begin{array}{l}\text { Constituent } \\
\text { ratio } \% \square \square\end{array}$ \\
\hline M.intracellulare & 30 & 39 & 32 & 49 & 97 & 87 & 334 & & 68.87 \\
\hline M.kansassi & 8 & 5 & 10 & 4 & 18 & 14 & 59 & & 12.16 \\
\hline M.abscessus & & 2 & 2 & 1 & 22 & 13 & 40 & & 8.25 \\
\hline M.fortuitum & 6 & 2 & & 2 & 3 & 2 & 15 & & 3.09 \\
\hline M.chelonae & 1 & 2 & 1 & 3 & 1 & 1 & 9 & & 1.85 \\
\hline M.avium & 1 & 1 & 1 & 1 & 1 & 1 & 6 & & 1.23 \\
\hline M.gordonae & & & & 1 & 1 & 3 & 5 & & 1.03 \\
\hline M.gilvum & 1 & & & & & 2 & 3 & & 0.61 \\
\hline M.szulgai & & & & & 2 & & 2 & & 0.41 \\
\hline M.smegmatis & & & 1 & & 1 & & 2 & & 0.41 \\
\hline M.peregrinum & & & & & & 2 & 2 & & 0.41 \\
\hline M.arupense & & & & 1 & & & 1 & & 0.21 \\
\hline M.mucogenicum & & & & & & 1 & 1 & & 0.21 \\
\hline M.marinum & & & & & & 1 & 1 & & 0.21 \\
\hline M.scrofulaceum & & & & & & 1 & 1 & & 0.21 \\
\hline M.shinjuku & & & & & & 1 & 1 & & 0.21 \\
\hline M.armeniacae & & & & & & 1 & 1 & & 0.21 \\
\hline M.porcinum & & & & & & 1 & 1 & & 0.21 \\
\hline M.neoaurum & & & & 1 & & & 1 & & 0.21 \\
\hline total & 47 & 51 & 47 & 62 & 147 & 131 & 485 & & $100 \%$ \\
\hline
\end{tabular}

$3 \otimes$ NTM drug susceptibility test results: 219 cases of NTM-PD were tested by microplate method. The results showed that NTM is generally high sensitivity to moxifloxacin, amikacin and clarithromycin $[100.00 \%$ (219/219), 96.80\% (212/219), 94.52\% (207/219), respectively), low sensitivity to imipenem/cilastatin, doxycycline, compound trimethoprim (2.74\% (6/219), 8.22\% (18/219), 9.59\% (21/219), respectively). (See Table 2).

Table 2219 cases of NTM-PD patients using microplate method to detect the sensitivity of different strains [ $n$ (sensitivity rate, \%)] 


\begin{tabular}{|c|c|c|c|c|c|c|}
\hline Drugs & M.intracellulare & M.kansassi & M.abscessus & M.fortuitum & others & total \\
\hline Linezolid & 25囚18.2】 & $23 \otimes 95.8 \rrbracket$ & 37ه100.0区 & 6冈6/7区 & $\begin{array}{l}10 \\
\square 71.4 \rrbracket\end{array}$ & 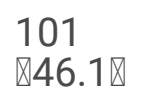 \\
\hline Clarithromycin & 133凶97.1区 & $23 \otimes 95.8 \rrbracket$ & $34 \llbracket 91.9 \rrbracket$ & $5 \otimes 5 / 7 \rrbracket$ & $\begin{array}{l}12 \\
\bigotimes 85.7 \rrbracket\end{array}$ & $\begin{array}{l}207 \\
\otimes 94.5 \rrbracket\end{array}$ \\
\hline Rifampicin & 136ه99.3凶 & $24 \rrbracket 100.0 \rrbracket$ & $5 \rrbracket 13.5 \rrbracket$ & $2 \rrbracket 2 / 7 \rrbracket$ & $\begin{array}{l}12 \\
\bigotimes 85.7 \rrbracket\end{array}$ & $\begin{array}{l}179 \\
\otimes 81.7 \rrbracket\end{array}$ \\
\hline Amikacin & $136 \llbracket 99.3 \rrbracket$ & $23 \otimes 95.8 \rrbracket$ & $34 \llbracket 91.9 \rrbracket$ & 7ه7/7区 & 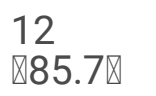 & $\begin{array}{l}212 \\
\square 96.8 \rrbracket\end{array}$ \\
\hline Imipenem & $5 \llbracket 3.6 \rrbracket$ & $0 \rrbracket 0.0 \rrbracket$ & $0 \rrbracket 0.0 \rrbracket$ & 1ه1/7区 & $0 \rrbracket 0.0 \rrbracket$ & $6 \rrbracket 2.7 \rrbracket$ \\
\hline Ethambutol & $66 \rrbracket 48.2 \rrbracket$ & $14 \rrbracket 58.3 \rrbracket$ & $0 \otimes 0.0 \rrbracket$ & $2 \varangle 2 / 7 \rrbracket$ & $7 \rrbracket 50.0 \rrbracket$ & $\begin{array}{l}89 \\
\square 40.6 \rrbracket\end{array}$ \\
\hline Cefoxitin & $129 \llbracket 94.2 \rrbracket$ & 4囚16.7凶 & $36 \varangle 97.3 \rrbracket$ & 6ख6/7ه & 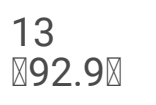 & $\begin{array}{l}188 \\
\varangle 85.8 \rrbracket\end{array}$ \\
\hline Azithromycin & $97 \otimes 70.8 \rrbracket$ & 20ه83.3》 & $22 \bowtie 59.5 \rrbracket$ & $2 \varangle 2 / 7 \rrbracket$ & $9 \llbracket 64.3 \rrbracket$ & 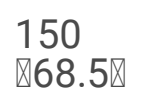 \\
\hline Rifabutin & 136ه99.3区 & $24 \rrbracket 100.0 \rrbracket$ & 20ه54.1》 & 7区7/7区 & $\begin{array}{l}10 \\
\text { ه100.0区 }\end{array}$ & 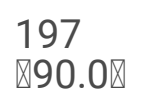 \\
\hline Tobramycin & $97 \otimes 70.8 \rrbracket$ & $7 \rrbracket 29.2 \rrbracket$ & $3 \otimes 8.1 \rrbracket$ & $0 \otimes 0.0 \otimes$ & $5 \llbracket 35.7 \rrbracket$ & 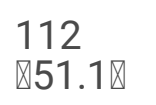 \\
\hline Gatifloxacin & $46 \rrbracket 33.6 \rrbracket$ & 18ه75.0ه & 6ख16.2】 & $7 \otimes 7 / 7 \rrbracket$ & $6 \varangle 42.0 \rrbracket$ & $\begin{array}{l}83 \\
\rrbracket 37.9 \rrbracket\end{array}$ \\
\hline Moxifloxacin & 137ه100.0区 & $24 \rrbracket 100.0 \rrbracket$ & 37囚100.0区 & $7 \otimes 7 / 7 \rrbracket$ & $\begin{array}{l}14 \\
\otimes 100.0 \rrbracket\end{array}$ & $\begin{array}{l}219 \\
\text { ه100.0区 }\end{array}$ \\
\hline Doxycycline & $6 \rrbracket 4.4 \rrbracket$ & 4冈16.7凶 & $3 \rrbracket 8.1 \rrbracket$ & 181/7区 & $4 \varangle 28.6 \rrbracket$ & $18 \bowtie 8.2 \rrbracket$ \\
\hline Minocycline & $5 \otimes 3.6 \rrbracket$ & $7 \rrbracket 29.2 \rrbracket$ & $5 \bigotimes 13.5 \rrbracket$ & $2 \varangle 2 / 7 \rrbracket$ & $4 \rrbracket 28.6 \rrbracket$ & $\begin{array}{l}23 \\
\square 10.5 \rrbracket\end{array}$ \\
\hline $\begin{array}{l}\text { Compound } \\
\text { Sulfamethoxazole }\end{array}$ & $4 \llbracket 2.9 \rrbracket$ & $7 \rrbracket 29.2 \rrbracket$ & $5 \bigotimes 13.5 \rrbracket$ & $2 \varangle 2 / 7 \rrbracket$ & $3 \llbracket 21.4 \rrbracket$ & $21 \otimes 9.6 \otimes$ \\
\hline
\end{tabular}

4. The treatment of patients in two groups $\otimes T$ This study showed that the bacteriological cure rate of the NTM-PD treatment regimen containing moxifloxacin was $73.68 \%$ (42/57), significantly higher than that of the regimen without moxifloxacin $(55.45 \%, 56 / 101)$, and the difference was statistically significant $(P<0.05)$. (See Table 3$)$.

Table3 Bacteriological cure rate distribution of patients in the two groups 


\begin{tabular}{|c|c|c|}
\hline group & $\begin{array}{l}\text { The number of } \\
\text { patients } \llbracket n \rrbracket\end{array}$ & bacteriological cure rate $₫ \mathrm{n} \otimes \% \bigotimes$ \\
\hline the moxifloxacin-containing regimen & 57 & 42刃73.68囚 \\
\hline the moxifloxacin-free regimen & 101 & $56 \rrbracket 55.45 \rrbracket$ \\
\hline$c^{2}$ & & 5.146 \\
\hline P-value & & 0.023 \\
\hline
\end{tabular}

\section{Discussion}

Recently, with the ageing of the population, the increase of immunosuppressive population, long-term use of antimicrobial drugs and advances in laboratory testing techniques, the incidence of reported NTM-PD has been increasing. The cell wall of NTM is highly hydrophobic and permeable, resulting in resistance to a variety of antimicrobial agents, and studies have shown that NTM generally has a high resistance rate to anti-TB drugs ${ }^{[6]}$. Existing in vitro drug sensitivity tests and clinical treatment often cannot be effectively linked, the lack of reliable drug sensitivity information is a very serious challenge to the clinical treatment of NTM-PD. Therefore, it is of great significance for the clinical treatment of NTM-PD to carry out accurate and rapid strain identification, reasonable drug sensitivity test and selection of effective drugs.

Because the distribution of NTM in nature is influenced by many factors, such as temperature and humidity, the distribution of NTM has regional differences. It is reported that the highest isolation rate of Mycobacterium avium complex in NTM isolates in the world is mainly from Australia. There is no significant difference between Europe and Asia, while North America is significantly less than the above two continents, and South Africa is the least ${ }^{[7]}$. Multicenter studies in China show that the main NTM in China is Mycobacterium abscess and Mycobacterium intracellular, which is the dominant strain in eastern China, and Mycobacterium abscess occupies an advantage in southern China ${ }^{[8]}$. Our province is located in the eastern part of China. This study showed that the main strains were M.intracellulare $₫ 68.87 \% \llbracket 334 / 485 \rrbracket$, which was consistent with the above reports.

In this study, 219 cases of NTM-PD strains were tested for drug sensitivity by microplate method. It is reported that microplate method drug susceptibility test is a new technique developed in recent years. The method can obtain more detailed and multi-antibiotic resistance data at the same time. It can also provide a concise judgment of drug sensitivity value with comparison. High coincidence rate and specificity, it has better clinical application value ${ }^{[5]}$. The results showed that all NTM strains had the highest sensitivity to moxifloxacin, which was $100.00 \%$ (219/219). Moxifloxacin can inhibit topoisomerase II and IV at the same time, so its drug resistance develops slowly, showing a low level of drug resistance. This is because drug resistance can occur only if the gene coding of two target enzymes in a pathogen is mutated simultaneously. Studies ${ }^{[9810]}$ have also shown that moxifloxacin has strong antibacterial activity in vitro, which is better than ethambutol, azithromycin and other first-line anti-NTM drugs. Meanwhile, this study showed that the cure rate of the 
regimen containing moxifloxacin was $73.68 \%(42 / 57)$, which was higher than that of the regimen without moxifloxacin $(55.45 \%, 56 / 101)$, and the difference was statistically significant $(P<0.05)$. Moxifloxacin reduces the possibility of drug interaction without cytochrome P450 enzyme metabolism, and the incidence of drug resistance is reduced when combined with other antibiotics. Some scholars believe that even if in vitro drug sensitivity test results show that moxifloxacin is resistant, but also recommended with clarithromycin, because moxifloxacin is convenient to take orally, has high absorption and availability, and patient compliance will be good, which is a better choice for clinical treatment ${ }^{[11]}$. Currently, there have been reports of long-term oral moxifloxacin therapy in patients with mycobacterium abscess complex pulmonary disease ${ }^{[12]}$.

In addition, the clinical application of moxifloxacin also has some limitations. Generally, moxifloxacin should not be used under the age of 18 because it affects chondrogenic development. At the same time, it should be attention that moxifloxacin can cause Q-T interval extension, mental symptoms and other adverse reactions. No serious adverse reactions such as anaphylactic shock, liver and kidney failure or cardiac arrest were found in this study. The limitations of this study are as follows: first, it is only the data of one hospital, and there is no multi-center demonstration; Second, the sample size is small, so it is necessary to increase the sample size for further demonstration. In conclusion, the drug susceptibility test of NTM-PD patients showed that all the strains were $100 \%$ sensitive to moxifloxacin, and the clinical cure rate was significantly improved. Therefore, moxifloxacin was recommended as a first-line drug for the treatment of NTM, which was worthy of clinical application and promotion.

\section{Declarations}

Ethics approval and consent to participate

All authors attest that they meet the Helsinki declaration. All methods were carried out in accordance with relevant guidelines and regulations. All experimental protocols were approved by the ethics committee of Shandong Public Health Clinical Center. Informed consent was waived by the Ethics Committee of (Shandong Public Health Clinical Center /IRB 2021XKYYEC-39) for this study due to retrospective nature.

Consent for publication

Not applicable

Availability of data and materials

The datasets used and/or analysed during the current study are available from the corresponding author on reasonable request. Because the materials contain the patient's name and other privacy, they cannot be disclosed.

Competing interest

The authors declare that they have no competing interest.

Funding 
Entry name:The 13th National five-year plan Science and Technology Major Project of China Project number:2018ZX10722301-001.

Authors' contributions

All authors attest that they meet the current International Committee of Medical Journal Editors (ICMJE) criteria for Authorship.

Acknowledgements

The authors gratefully acknowledge the financial supports by the Foundation of The 13th National five-year plan Science and Technology Major Project of China.

\section{References}

1. Daley CL, laccarino JM, Lange C, et al. Treatment of nontuberculous mycobacterial pulmonary disease: an official ATS/ERS/ESCMID/IDSA clinical practice guideline [J]. Eur Respir J, 2020, 56(1): 200053.

DOI:org/10.1183 13993003.00535-2020.

2. Furuuchi K, Morimoto K, Yoshiyama T, et al. Interrelational changes in the epidemiology and clinical features of nontuberculous mycobacterial pulmonary disease and tuberculosis in a referral hospital in Japan[J]. Respir med, 2019, 152:74-80. Dol: 10.1016/j.rmed.2019.05.001.

3. Winthrop KL, Marras TK, Adjemian J, et al. Incidence and Prevalence of Nontuberculous Mycobacterial Lung Disease in a Large United States Managed Care Health Plan, 2008-2015[J]. Ann Am Thorac Soc, 2020, 17 (2):178-185. DOI: 10.1513 / AnnalsATS.201804-2360C.

4. Chinese Medical Association Tuberculosis Credit Association. Guidelines for the Diagnosis and Treatment of Nontuberculous Mycobacteria Disease (2020 Edition) [J]. Chinese Journal of Tuberculosis and Breathing, 2020, 43(11): 918-946..doi:10.3760/cma.j.cn112147-20200508 -00570.

5. Li Jing, Wang Zhicun, Bai Guanghong, et al. The clinical value of MicroDST-(TM) microporous plate assay for anti-tuberculosis first-and second-line drug sensitivity tests [J], China Journal of Lung Disease (Electronic Edition),2018, 11(5): 583-587,doi:10.3877/ cma.j.issn.1674-6902.2018.05.014.

6. Zhang Jie, Su Jianrong, Ding Beichuan, et al. Study on the distribution and drug resistance of Nontuberculous Mycobacteria in Beijing [J]. Chinese Journal of Tuberculosis and Breathing, 2017,40(3):210-214. Dol:10.3760/cma.j.issn. 1001-0939. 2017.03 .013.

7. Hoefsloot W, van Ingen J, Andrejak C, et al. The geographic diversity of nontuberculous mycobacteria isolated from pulmonary samples: an NTM-NET collaborative study[J]. Eur Respir J,2013, 42 (6) : 16041613. doi:10.1183/09031936.0 0149212.

8. Pang Y, Tan Y, Chen J, et al. Diversity of nontuberculous mycobacteria in eastern and southern China: a cross-sectional study [J]. Eur Respir J, 2017, 49(3) : 1601429. doi:10.1183/13993003.01429-2016.

9. Zhang Z, Pang Y, Wang Y, et al. Differences in risk factors and drug susceptibility between Mycobacterium avium and Mycobacterium intracellulare lung diseases in China[J]. Int J Antimicrob Agents, 2015, 45(5): 491-495. doi:10.1016/j.ijantimicag. 2015. 01.012. 
10. Wei G, Huang M, Wang G, et al. Antimicrobial susceptibility testing and genotyping of Mycobacterium avium isolates of two tertiary tuberculosis designated hospital, China[J]. Infect Genet Evol, 2015, 36: 141146. doi:10.1016/j.meegid. 2015.09. 015.

11. Wu Yue, Deng Xizi, Hu Fengyu, et al. Pathogenic spectrum, clinical characteristics and drug resistance of aids combined with Nontuberculous Mycobacteria lung disease[J]. Chinese Journal of Infectious Diseases, 2017, 35(3):142-145. doi:10.3760/cma.j.issn.1000 -6680.2017.03.004.

12. Koh WJ, Jeon K, Shin SJ. Successful treatment of Mycobacterium massiliense lung disease with oral antibiotics only[J]. Antimicrob Agents Chemother, 2013, 57(2): 1098-1100. doi: 10.1128/AAC. 02016-12.

\section{Supplementary Files}

This is a list of supplementary files associated with this preprint. Click to download.

- rawdata.xlsx 